involvements of gastrointestinal tract. (3)The endoscopic features and diagnostic rate: 258 patients had complete endoscopic profiles, and the endoscopic subtypes were ulcer type, $46.5 \%$, diffuse infiltration, $27.1 \%$, uplift the lump, $17.4 \%$ and superficial lesions, $8.9 \%$ (figure 1 ). Of these, 131 underwent one or more endoscopic biopsies or EMR/ESD to clarify the pathological diagnosis. However, the endoscopic diagnostic rate was significantly different among different lesions: esophagus (1/2,50\%), stomach (90/ $182,49.5 \%)$, duodenum and small intestine $(16 / 63,25.4 \%)$, and colon (24/88,27.3\%). (4)Diagnostic method: 131 cases $(42.0 \%)$ were confirmed by endoscopic diagnosis, and the rest were confirmed by surgical pathology $(158 / 312,50.6 \%)$ or puncture pathology (23/312,7.3\%). (5)Prognostic factors: The median follow-up time of the patients was 36.9 months (range: $0.2-317.4$ ), and the three-year progression-free survival rate and overall survival rate were $73.6 \%$ and $78.7 \%$ respectively. Multivariate analysis showed that IPI and B symptom were independent adverse prognostic factors $(\mathrm{P}<0.05)$.

Conclusions PGI-DLBCL does not have specific clinical symptoms. The most common site of lesion is the stomach, but the whole digestive tract can be involved. The diagnosis of this disease is mainly by endoscopy or surgical pathology, and the endoscopic diagnostic rate is affected by the lesion site. The prognosis is poor, and increased IPI and B symptoms suggest a poor prognosis. Surgery cannot improve the overall survival of patients.

\section{IDDF2019-ABS-0156 DECREASED SERUM ACYLATED GHRELIN LEVEL WITH THE EXTENT OF OLGA AND OLGIM STAGES IN DYSPEPTIC PATIENTS}

${ }^{1}$ Wai Phyo Lwin*, ${ }^{2}$ Aye Min Soe, ${ }^{3}$ Phyo Wai Kyaw, ${ }^{4}$ Tin Moe Wai. ${ }^{1}$ Department of Gastroenterology,No; ${ }^{2}$ Military Hospital; ${ }^{500}$ Bedded), Yangon, Burma; ${ }^{2}$ Department of Gastroenterology and Hepatobiliary Medicine, Defence Services Medical Academy, Yangon, Burma; ${ }^{3}$ Department of Pathology,No; ${ }^{2}$ Military Hospital; ${ }^{500}$ Bedded), Yangon, Burma; ${ }^{4}$ Department of Gastroenterology, Yangon General Hospital, Yangon, Burma

\subsection{6/gutjnl-2019-IDDFabstracts.171}

Background Serum acylated ghrelin is mainly produced from oxyntic mucosa of the stomach and its production is impaired in the premalignant condition of gastric cancer like atrophy and intestinal metaplasia. This study was done to compare serum acylated ghrelin level to stages of operative link for gastritis assessment (atrophy) (OLGA) and operative link for gastritis intestinal metaplasia (OLGIM) in dyspeptic patients.

Methods This study was conducted in sixty-seven dyspeptic patients at $\mathrm{No}(2)$ Military Hospital(500 bedded) from December 2017 to September 2018. Updated Sydney system was used for histology report and the results were staged according to OLGA and OLGIM system. Fasting acylated ghrelin level was measured by enzyme-linked immunosorbent assay (ELISA) and it was compared between the stages of OLGA and OLGIM by using Kruskal Wallis method.

Results Out of 67 patients, $44(65.7 \%)$ were stage 0,11 (16.4\%) were stage I and $12(17.9 \%)$ were stage II \& above for OLGA staging. Median acylated ghrelin level was lowest in OLGA stage II $\&$ above and highest in OLGA stage 0 ( 364.0 vs $818.0 \mathrm{pg} / \mathrm{ml}, \mathrm{p}<0.001)$. Post hoc analysis showed a significant difference between stage 0 and stage II $\&$ above but no significant difference between stage 0 and stage I, stage I and stage II \& above. Among 67 patients, 46 (68.7\%) were stage $0,17(25.4 \%)$ were stage I and $4(6.0 \%)$ were stage II $\&$ above for OLGIM staging. Median acylated ghrelin level was lowest in OLGIM stage II \& above and highest in OLGIM stage 0 (181.1vs $783.1 \mathrm{pg} / \mathrm{ml}, \mathrm{p}=0.004)$.Post hoc analysis showed there was a significant difference between stage 0 and stage II $\&$ above but no significant difference between stage 0 and stage I, stage I and stage II $\&$ above. The optimum cut off value for detection of antrum atrophy was $342.16 \mathrm{pg} / \mathrm{ml}$ with the sensitivity $73 \%$ and specificity $72 \%$ and for corpus atrophy was $316.21 \mathrm{pg} / \mathrm{ml}$ with the sensitivity and specificity of $64 \%$.

Conclusions Serum acylated ghrelin level decreased in parallel with the extent of gastric atrophy and intestinal metaplasia. It may be useful as a non-invasive marker for screening of premalignant condition in dyspeptic patients.

Abstract IDDF2019-ABS-0156 Figure 1 Roc curve for outcome antrum atrophy using acylated ghrelin \& corpus atrophy using acylated ghrelin levels 